\title{
SUPPLEMENTARY MATERIALS TO "A TWO-STEP APPROACH TO MODEL PRECIPITATION EXTREMES IN CALIFORNIA BASED ON MAX-STABLE AND MARGINAL POINT PROCESSES"
}

\author{
By Hongwei Shang*, Jun Yan ${ }^{\dagger}$ And Xuebin Zhang ${ }^{\ddagger}$ \\ Hewlett Packard Labs* ${ }^{*}$ University of Connecticut ${ }^{\dagger}$ and Environment \\ Canada $a^{\ddagger}$
}

1. Sandwich Variance Estimator. The slope matrix $A$ can be estimated with

$$
A_{n}=\frac{1}{n} \sum_{t=1}^{n}\left(\begin{array}{cc}
\partial \psi_{1 t}\left(\hat{\beta}_{n}\right) / \partial \beta^{\top} & 0 \\
\partial \psi_{2 t}\left(\hat{\beta}_{n}, \hat{\theta}_{n}\right) / \partial \beta^{\top} & \partial \psi_{2 t}\left(\hat{\beta}_{n}, \hat{\theta}_{n}\right) / \partial \theta^{\top}
\end{array}\right),
$$

where the triangular form comes from the fact that the first step does not involve the dependence parameters. This matrix involves the second-order derivatives of the composite loglikelihood, which can be difficult to obtain in practice. An alternative formula that involves only the first-order derivatives of the composite loglikelihoods can be derived. We first look into the contribution of each site and each pair at each year to the terms in $A_{n}$. Let $\psi_{1 t, s}(\beta)=\partial \ell_{1 t, s} / \partial \beta$. Let $\ell_{2 t,(i, j)}(\theta, \beta)=\log f_{i j t}\left(\left(M_{i, t}, M_{j, t}\right) ; \theta, \beta\right)$ and $\psi_{2 t,(i, j)}(\beta, \theta)=\partial \ell_{2 t,(i, j)} / \partial \theta$. Then, $A_{n}$ can be rewritten as

$$
A_{n}=\frac{1}{n} \sum_{t=1}^{n}\left(\begin{array}{c}
\sum_{i=1}^{S} \partial \psi_{1 t, s}\left(\hat{\beta}_{n}\right) / \partial \beta^{\top} \\
\sum_{i<j}^{S} \partial \psi_{2 t,(i, j)}\left(\hat{\beta}_{n}, \hat{\theta}_{n}\right) / \partial \beta^{\top} \quad \sum_{i<j}^{S} \partial \psi_{2 t,(i, j)}\left(\hat{\beta}_{n}, \hat{\theta}_{n}\right) / \partial \theta^{\top}
\end{array}\right) .
$$

Instead of calculating the second-order derivatives in $A_{n}$, we use the firstorder derivatives based on the second Bartlett identity, assuming that the univariate and bivariate marginal models are correctly specified. Let $\phi_{2 t,(i, j)}(\beta, \theta)=$ $\partial \ell_{2 t,(i, j)} / \partial \beta$. We can estimate $A$ by

$$
\hat{A}_{n}=-\frac{1}{n} \sum_{t=1}^{n}\left(\begin{array}{c}
\sum_{i=1}^{S} \psi_{1 t, s}\left(\hat{\beta}_{n}\right) \psi_{1 t, s}^{\top}\left(\hat{\beta}_{n}\right) \\
\sum_{i<j}^{S} \psi_{2 t,(i, j)}\left(\hat{\beta}_{n}, \hat{\theta}_{n}\right) \phi_{2 t,(i, j)}^{\top}\left(\hat{\beta}_{n}, \hat{\theta}_{n}\right) \quad \sum_{i<j}^{S} \psi_{2 t,(i, j)}\left(\hat{\beta}_{n}, \hat{\theta}_{n}\right) \psi_{2 t,(i, j)}^{\top}\left(\hat{\beta}_{n}, \hat{\theta}_{n}\right)
\end{array}\right) .
$$

22 For $B$, we estimate it with

$$
B_{n}=\frac{1}{n} \sum_{t=1}^{n}\left(\begin{array}{cc}
\psi_{1 t}\left(\hat{\beta}_{n}\right) \psi_{1 t}^{\top}\left(\hat{\beta}_{n}\right) & \psi_{1 t}\left(\hat{\beta}_{n}\right) \psi_{2 t}^{\top}\left(\hat{\beta}_{n}, \hat{\theta}_{n}\right) \\
\psi_{2 t}\left(\hat{\beta}_{n}, \hat{\theta}_{n}\right) \psi_{1 t}^{\top}\left(\hat{\beta}_{n}\right) & \psi_{2 t}\left(\hat{\beta}_{n}, \hat{\theta}_{n}\right) \psi_{2 t}^{\top}\left(\hat{\beta}_{n}, \hat{\theta}_{n}\right)
\end{array}\right)
$$


The estimation of $B$ in this problem is straightforward because we have replicates, unlike in the usual spatial data setting where there is only one replicate and some bootstrap procedure would need to be developed (Heagerty and Lele, 1998; Heagerty and Lumley, 2000).

The sandwich estimator of $\Omega$ is then $\hat{\Omega}_{n}=A_{n}^{-1} B_{n}\left(A_{n}^{-1}\right)^{\top}$.

\section{More Results from the Simulation Study.}

\subsection{Simulation from Smith, Schlather, and Geometric Gaussian Model.}

2.1.1. Bias, Variation, and Empirical Coverage. To validate the twostep approach, we summarize the bias, the empirical standard error (ESE), the average of the standard error (ASE) from the sandwich variance estimator, and the empirical coverage percentage of the $95 \%$ confidence intervals based on 1000 replicates in Table 1 for the Smith model, Table 2 for the Schlather model, and Table 3 for the geometric Gaussian model. Only results for $n \in\{50,100\}$ are reported.

2.1.2. Relative Efficiency in Estimating Return Levels. Table 4 summarizes the relative efficiency of the pairwise likelihood approach using block maxima only with the two-step approach as the reference in estimating joint and individual return levels. The three sites used were $s_{1}=(10,10)$, $s_{2}=(10,11)$ and $s_{3}=(10,0)$. The pairs $\left(s_{1}, s_{2}\right)$ and $\left(s_{1}, s_{3}\right)$ represent pairs with short and long distance apart, respectively.

2.2. Simulation from the Max-Domain of Attraction of Extremal-t Process.

2.2.1. Details of Data Generation. The purpose of this simulation study was to investigate the properties of the two-step estimator with data generated from not the limiting model but in its max-domain of attraction. The study region, the marginal distribution of block maxima, the number of sites $S$, the sample size $n$ and the block size $m$ are all defined the same as in the Simulation section. The $t$ process in the max-domain of the extremal- $t$ process can be constructed as $\sqrt{Y} W$, where $Y^{-1}$ is a $\Gamma(\nu / 2,2 / n u)$ variate with mean 1 , and $W$ is a stationary Gaussian process with correlation function $\rho$. The extremal- $t$ process is characterized by the degree of freedom (d.f.) $\nu$ and the dispersion function $\rho$ (the correlation function of $W$ ). We need to generate data such that as $m \rightarrow \infty, 1)$ the limiting marginal distribution of block maxima at each site $s$ is GEV with parameters $\left(\mu_{s}, \sigma_{s}, \xi_{s}\right)$; and 2$)$ the limiting copula is the extremal- $t$ copula characterized by $\nu$ and $\rho(\cdot \mid \theta)$, where $\rho$ is an exponential correlation function with range parameter $\theta=\alpha$. 
TABLE 1

Bias, empirical standard errors (ESE), average standard errors (ASE) from the sandwich estimator, and empirical coverage percentage (CP) of $95 \%$ confidence intervals for the model parameters (Par) from the two-step approach based on 1000 replicates for the Smith model.

\begin{tabular}{|c|c|c|c|c|c|c|c|c|c|c|c|}
\hline \multirow[b]{2}{*}{ Dep } & \multirow[b]{2}{*}{$S$} & \multirow[b]{2}{*}{ Par } & \multirow[b]{2}{*}{ True } & \multicolumn{4}{|c|}{$n=50$} & \multicolumn{4}{|c|}{$n=100$} \\
\hline & & & & bias & ESE & ASE & $\mathrm{CP}$ & bias & ESE & ASE & $\mathrm{CP}$ \\
\hline \multirow[t]{16}{*}{$\mathrm{W}$} & \multirow[t]{8}{*}{25} & $\tau$ & 20.00 & 0.024 & 2.190 & 2.046 & 92.1 & 0.019 & 1.562 & 1.492 & 93.5 \\
\hline & & $\beta_{\mu, 0}$ & 15.00 & -0.020 & 0.214 & 0.212 & 94.2 & -0.007 & 0.151 & 0.150 & 95.0 \\
\hline & & $\beta_{\mu, 1}$ & -0.20 & -0.000 & 0.012 & 0.013 & 95.8 & -0.000 & 0.009 & 0.009 & 95.0 \\
\hline & & $\beta_{\mu, 2}$ & 0.25 & -0.001 & 0.014 & 0.014 & 94.6 & -0.000 & 0.009 & 0.010 & 95.4 \\
\hline & & $\beta_{\sigma, 0}$ & 4.00 & 0.015 & 0.176 & 0.168 & 93.7 & 0.020 & 0.120 & 0.119 & 95.4 \\
\hline & & $\beta_{\sigma, 1}$ & -0.04 & -0.000 & 0.006 & 0.007 & 95.5 & -0.000 & 0.005 & 0.005 & 94.2 \\
\hline & & $\beta_{\sigma, 2}$ & 0.08 & 0.000 & 0.008 & 0.007 & 93.8 & 0.000 & 0.005 & 0.005 & 95.8 \\
\hline & & $\beta_{\xi, 0}$ & 0.20 & -0.007 & 0.022 & 0.021 & 92.5 & -0.007 & 0.015 & 0.015 & 91.5 \\
\hline & \multirow[t]{8}{*}{50} & $\tau$ & 20.00 & -0.065 & 1.914 & 1.760 & 90.9 & -0.028 & 1.315 & 1.268 & 93.4 \\
\hline & & $\beta_{\mu, 0}$ & 15.00 & -0.003 & 0.205 & 0.203 & 95.1 & -0.007 & 0.142 & 0.143 & 94.1 \\
\hline & & $\beta_{\mu, 1}$ & -0.20 & 0.001 & 0.011 & 0.012 & 95.4 & -0.000 & 0.008 & 0.008 & 95.2 \\
\hline & & $\beta_{\mu, 2}$ & 0.25 & -0.000 & 0.012 & 0.012 & 94.6 & -0.000 & 0.009 & 0.009 & 94.7 \\
\hline & & $\beta_{\sigma, 0}$ & 4.00 & 0.020 & 0.170 & 0.160 & 93.5 & 0.017 & 0.114 & 0.113 & 95.4 \\
\hline & & $\beta_{\sigma, 1}$ & -0.04 & 0.000 & 0.006 & 0.006 & 95.2 & -0.000 & 0.004 & 0.004 & 94.4 \\
\hline & & $\beta_{\sigma, 2}$ & 0.08 & 0.000 & 0.007 & 0.007 & 93.7 & 0.000 & 0.005 & 0.005 & 96.0 \\
\hline & & $\beta_{\xi, 0}$ & 0.20 & -0.007 & 0.021 & 0.020 & 89.1 & -0.007 & 0.014 & 0.014 & 91.2 \\
\hline \multirow[t]{16}{*}{ M } & \multirow[t]{8}{*}{25} & $\tau$ & 200.00 & 2.804 & 41.217 & 39.774 & 93.0 & 1.536 & 29.549 & 28.238 & 93.6 \\
\hline & & $\beta_{\mu, 0}$ & 15.00 & -0.029 & 0.367 & 0.393 & 95.6 & -0.014 & 0.275 & 0.275 & 94.2 \\
\hline & & $\beta_{\mu, 1}$ & -0.20 & 0.001 & 0.013 & 0.014 & 95.4 & 0.000 & 0.009 & 0.010 & 96.0 \\
\hline & & $\beta_{\mu, 2}$ & 0.25 & -0.000 & 0.014 & 0.015 & 96.2 & -0.001 & 0.011 & 0.011 & 94.3 \\
\hline & & $\beta_{\sigma, 0}$ & 4.00 & 0.009 & 0.308 & 0.317 & 94.2 & 0.021 & 0.231 & 0.222 & 95.1 \\
\hline & & $\beta_{\sigma, 1}$ & -0.04 & 0.000 & 0.007 & 0.008 & 95.6 & -0.000 & 0.005 & 0.005 & 95.7 \\
\hline & & $\beta_{\sigma, 2}$ & 0.08 & 0.000 & 0.009 & 0.010 & 95.5 & 0.000 & 0.007 & 0.007 & 94.4 \\
\hline & & $\beta_{\xi, 0}$ & 0.20 & -0.008 & 0.040 & 0.041 & 93.8 & -0.006 & 0.030 & 0.029 & 92.1 \\
\hline & \multirow[t]{8}{*}{50} & $\tau$ & 200.00 & 1.706 & 41.257 & 38.645 & 90.7 & 1.879 & 28.250 & 27.847 & 92.8 \\
\hline & & $\beta_{\mu, 0}$ & 15.00 & -0.027 & 0.389 & 0.389 & 94.2 & -0.003 & 0.274 & 0.275 & 95.1 \\
\hline & & $\beta_{\mu, 1}$ & -0.20 & 0.001 & 0.014 & 0.013 & 92.7 & -0.000 & 0.009 & 0.009 & 95.5 \\
\hline & & $\beta_{\mu, 2}$ & 0.25 & -0.002 & 0.015 & 0.015 & 94.9 & -0.000 & 0.011 & 0.011 & 94.0 \\
\hline & & $\beta_{\sigma, 0}$ & 4.00 & -0.001 & 0.323 & 0.312 & 92.4 & 0.025 & 0.218 & 0.221 & 95.9 \\
\hline & & $\beta_{\sigma, 1}$ & -0.04 & 0.000 & 0.008 & 0.007 & 94.4 & -0.001 & 0.005 & 0.005 & 96.1 \\
\hline & & $\beta_{\sigma, 2}$ & 0.08 & -0.001 & 0.009 & 0.009 & 95.3 & 0.000 & 0.007 & 0.007 & 94.3 \\
\hline & & $\beta_{\xi, 0}$ & 0.20 & -0.010 & 0.042 & 0.040 & 90.5 & -0.007 & 0.028 & 0.028 & 94.9 \\
\hline \multirow[t]{16}{*}{$\mathrm{S}$} & \multirow[t]{8}{*}{25} & $\tau$ & 2000.00 & 42.735 & 474.266 & 503.661 & 95.0 & 11.821 & 341.721 & 345.869 & 93.9 \\
\hline & & $\beta_{\mu, 0}$ & 15.00 & -0.019 & 0.458 & 0.476 & 95.7 & -0.031 & 0.318 & 0.329 & 94.5 \\
\hline & & $\beta_{\mu, 1}$ & -0.20 & 0.000 & 0.009 & 0.009 & 95.1 & 0.000 & 0.006 & 0.006 & 95.6 \\
\hline & & $\beta_{\mu, 2}$ & 0.25 & -0.001 & 0.011 & 0.012 & 94.5 & -0.001 & 0.008 & 0.008 & 95.4 \\
\hline & & $\beta_{\sigma, 0}$ & 4.00 & 0.014 & 0.376 & 0.397 & 94.9 & 0.006 & 0.269 & 0.272 & 94.7 \\
\hline & & $\beta_{\sigma, 1}$ & -0.04 & -0.000 & 0.006 & 0.006 & 95.1 & -0.000 & 0.004 & 0.004 & 96.2 \\
\hline & & $\beta_{\sigma, 2}$ & 0.08 & 0.000 & 0.009 & 0.009 & 94.2 & 0.000 & 0.006 & 0.006 & 95.6 \\
\hline & & $\beta_{\xi, 0}$ & 0.20 & -0.008 & 0.052 & 0.056 & 95.1 & -0.008 & 0.038 & 0.038 & 94.9 \\
\hline & \multirow[t]{8}{*}{50} & $\tau$ & 2000.00 & 43.752 & 485.566 & 504.363 & 93.8 & 36.521 & 348.386 & 347.800 & 94.4 \\
\hline & & $\beta_{\mu, 0}$ & 15.00 & -0.025 & 0.451 & 0.479 & 94.8 & -0.005 & 0.331 & 0.330 & 95.4 \\
\hline & & $\beta_{\mu, 1}$ & -0.20 & 0.000 & 0.008 & 0.009 & 95.2 & -0.000 & 0.006 & 0.006 & 95.8 \\
\hline & & $\beta_{\mu, 2}$ & 0.25 & -0.001 & 0.011 & 0.012 & 95.0 & -0.000 & 0.008 & 0.008 & 95.2 \\
\hline & & $\beta_{\sigma, 0}$ & 4.00 & 0.009 & 0.378 & 0.401 & 94.4 & 0.022 & 0.271 & 0.273 & 94.7 \\
\hline & & $\beta_{\sigma, 1}$ & -0.04 & -0.000 & 0.005 & 0.006 & 95.6 & -0.000 & 0.004 & 0.004 & 95.4 \\
\hline & & $\beta_{\sigma, 2}$ & 0.08 & 0.000 & 0.009 & 0.009 & 94.6 & 0.000 & 0.006 & 0.006 & 94.3 \\
\hline & & $\beta_{\xi, 0}$ & 0.20 & -0.009 & 0.053 & 0.056 & 95.0 & -0.006 & 0.036 & 0.038 & 95.5 \\
\hline
\end{tabular}


TABle 2

Bias, empirical standard errors (ESE), average standard errors (ASE) from the sandwich estimator, and empirical coverage percentage (CP) of $95 \%$ confidence intervals for the model parameters (Par) from the two-step approach based on 1000 replicates for the Schlather model.

\begin{tabular}{|c|c|c|c|c|c|c|c|c|c|c|c|}
\hline \multirow[b]{2}{*}{ Dep } & \multirow[b]{2}{*}{$S$} & \multirow[b]{2}{*}{ Par } & \multirow[b]{2}{*}{ True } & \multicolumn{4}{|c|}{$n=50$} & \multicolumn{4}{|c|}{$n=100$} \\
\hline & & & & bias & ESE & ASE & $\mathrm{CP}$ & bias & $\mathrm{ESE}$ & ASE & $\mathrm{CP}$ \\
\hline \multirow[t]{16}{*}{ W } & \multirow[t]{8}{*}{25} & $\alpha$ & 5.20 & 0.052 & 1.480 & 1.422 & 93.1 & 0.014 & 1.065 & 1.027 & 93.7 \\
\hline & & $\beta_{\mu, 0}$ & 15.00 & -0.021 & 0.305 & 0.312 & 94.3 & -0.011 & 0.209 & 0.220 & 96.0 \\
\hline & & $\beta_{\mu, 1}$ & -0.20 & 0.000 & 0.008 & 0.008 & 95.0 & 0.000 & 0.006 & 0.006 & 96.7 \\
\hline & & $\beta_{\mu, 2}$ & 0.25 & -0.000 & 0.010 & 0.010 & 95.8 & -0.000 & 0.007 & 0.007 & 96.0 \\
\hline & & $\beta_{\sigma, 0}$ & 4.00 & 0.007 & 0.254 & 0.252 & 94.4 & 0.012 & 0.178 & 0.178 & 95.1 \\
\hline & & $\beta_{\sigma, 1}$ & -0.04 & 0.000 & 0.005 & 0.005 & 94.8 & -0.000 & 0.003 & 0.003 & 95.5 \\
\hline & & $\beta_{\sigma, 2}$ & 0.08 & 0.000 & 0.007 & 0.007 & 94.1 & 0.000 & 0.005 & 0.005 & 95.1 \\
\hline & & $\beta_{\xi, 0}$ & 0.20 & -0.009 & 0.033 & 0.033 & 92.8 & -0.008 & 0.024 & 0.023 & 91.9 \\
\hline & \multirow[t]{8}{*}{50} & $\alpha$ & 5.20 & -0.009 & 1.356 & 1.292 & 92.0 & 0.023 & 0.984 & 0.938 & 93.9 \\
\hline & & $\beta_{\mu, 0}$ & 15.00 & -0.016 & 0.303 & 0.306 & 94.5 & -0.007 & 0.213 & 0.217 & 95.3 \\
\hline & & $\beta_{\mu, 1}$ & -0.20 & 0.000 & 0.007 & 0.007 & 95.0 & 0.000 & 0.005 & 0.005 & 95.3 \\
\hline & & $\beta_{\mu, 2}$ & 0.25 & -0.001 & 0.009 & 0.009 & 95.4 & -0.000 & 0.006 & 0.006 & 94.6 \\
\hline & & $\beta_{\sigma, 0}$ & 4.00 & 0.001 & 0.246 & 0.248 & 95.3 & 0.017 & 0.179 & 0.176 & 95.7 \\
\hline & & $\beta_{\sigma, 1}$ & -0.04 & 0.000 & 0.004 & 0.004 & 94.5 & -0.000 & 0.003 & 0.003 & 95.8 \\
\hline & & $\beta_{\sigma, 2}$ & 0.08 & -0.000 & 0.006 & 0.006 & 94.6 & 0.000 & 0.004 & 0.004 & 94.8 \\
\hline & & $\beta_{\xi, 0}$ & 0.20 & -0.010 & 0.032 & 0.032 & 92.7 & -0.008 & 0.024 & 0.023 & 92.9 \\
\hline \multirow[t]{16}{*}{ M } & \multirow[t]{8}{*}{25} & $\alpha$ & 24.30 & 0.107 & 5.675 & 5.660 & 92.1 & -0.004 & 4.255 & 4.030 & 92.3 \\
\hline & & $\beta_{\mu, 0}$ & 15.00 & -0.017 & 0.356 & 0.376 & 95.1 & -0.017 & 0.248 & 0.264 & 94.8 \\
\hline & & $\beta_{\mu, 1}$ & -0.20 & 0.000 & 0.010 & 0.010 & 95.9 & 0.000 & 0.007 & 0.007 & 94.9 \\
\hline & & $\beta_{\mu, 2}$ & 0.25 & -0.000 & 0.011 & 0.012 & 95.9 & -0.001 & 0.008 & 0.009 & 96.4 \\
\hline & & $\beta_{\sigma, 0}$ & 4.00 & 0.009 & 0.309 & 0.307 & 93.5 & 0.014 & 0.206 & 0.215 & 95.3 \\
\hline & & $\beta_{\sigma, 1}$ & -0.04 & 0.000 & 0.006 & 0.006 & 95.2 & -0.000 & 0.004 & 0.004 & 95.8 \\
\hline & & $\beta_{\sigma, 2}$ & 0.08 & 0.000 & 0.008 & 0.008 & 95.6 & 0.000 & 0.005 & 0.006 & 95.7 \\
\hline & & $\beta_{\xi, 0}$ & 0.20 & -0.009 & 0.043 & 0.041 & 91.4 & -0.007 & 0.028 & 0.029 & 93.8 \\
\hline & \multirow[t]{8}{*}{50} & $\alpha$ & 24.30 & -0.181 & 5.792 & 5.414 & 92.7 & 0.098 & 4.092 & 3.911 & 93.9 \\
\hline & & $\beta_{\mu, 0}$ & 15.00 & -0.031 & 0.361 & 0.372 & 95.5 & -0.008 & 0.264 & 0.263 & 94.3 \\
\hline & & $\beta_{\mu, 1}$ & -0.20 & 0.001 & 0.009 & 0.010 & 95.0 & 0.000 & 0.006 & 0.007 & 96.0 \\
\hline & & $\beta_{\mu, 2}$ & 0.25 & -0.001 & 0.011 & 0.012 & 95.2 & -0.000 & 0.008 & 0.008 & 94.7 \\
\hline & & $\beta_{\sigma, 0}$ & 4.00 & 0.000 & 0.303 & 0.301 & 94.5 & 0.020 & 0.214 & 0.213 & 94.5 \\
\hline & & $\beta_{\sigma, 1}$ & -0.04 & 0.000 & 0.005 & 0.006 & 95.1 & -0.000 & 0.004 & 0.004 & 95.4 \\
\hline & & $\beta_{\sigma, 2}$ & 0.08 & -0.000 & 0.007 & 0.008 & 95.0 & 0.000 & 0.005 & 0.005 & 95.7 \\
\hline & & $\beta_{\xi, 0}$ & 0.20 & -0.010 & 0.041 & 0.040 & 91.6 & -0.007 & 0.028 & 0.028 & 93.3 \\
\hline \multirow[t]{16}{*}{$\mathrm{S}$} & \multirow[t]{8}{*}{25} & $\alpha$ & 242.90 & -2.597 & 61.013 & 62.507 & 93.1 & -0.292 & 41.894 & 44.078 & 94.7 \\
\hline & & $\beta_{\mu, 0}$ & 15.00 & -0.034 & 0.446 & 0.464 & 95.2 & -0.026 & 0.309 & 0.322 & 95.0 \\
\hline & & $\beta_{\mu, 1}$ & -0.20 & 0.000 & 0.007 & 0.008 & 95.0 & 0.001 & 0.005 & 0.005 & 95.7 \\
\hline & & $\beta_{\mu, 2}$ & 0.25 & -0.001 & 0.011 & 0.011 & 94.3 & -0.000 & 0.008 & 0.008 & 96.0 \\
\hline & & $\beta_{\sigma, 0}$ & 4.00 & -0.015 & 0.365 & 0.385 & 94.5 & 0.002 & 0.247 & 0.266 & 96.1 \\
\hline & & $\beta_{\sigma, 1}$ & -0.04 & 0.000 & 0.005 & 0.005 & 94.9 & 0.000 & 0.003 & 0.004 & 95.8 \\
\hline & & $\beta_{\sigma, 2}$ & 0.08 & -0.000 & 0.008 & 0.009 & 94.8 & 0.000 & 0.006 & 0.006 & 95.7 \\
\hline & & $\beta_{\xi, 0}$ & 0.20 & -0.013 & 0.051 & 0.055 & 94.2 & -0.009 & 0.035 & 0.038 & 95.3 \\
\hline & \multirow[t]{8}{*}{50} & $\alpha$ & 242.90 & 5.419 & 63.473 & 63.749 & 94.0 & 1.940 & 43.403 & 43.832 & 94.5 \\
\hline & & $\beta_{\mu, 0}$ & 15.00 & 0.015 & 0.460 & 0.470 & 95.0 & -0.012 & 0.308 & 0.323 & 95.9 \\
\hline & & $\beta_{\mu, 1}$ & -0.20 & -0.000 & 0.007 & 0.008 & 96.2 & 0.000 & 0.005 & 0.005 & 95.7 \\
\hline & & $\beta_{\mu, 2}$ & 0.25 & 0.000 & 0.011 & 0.011 & 95.0 & -0.000 & 0.008 & 0.008 & 95.0 \\
\hline & & $\beta_{\sigma, 0}$ & 4.00 & 0.036 & 0.374 & 0.391 & 94.9 & 0.016 & 0.257 & 0.266 & 94.4 \\
\hline & & $\beta_{\sigma, 1}$ & -0.04 & -0.001 & 0.005 & 0.005 & 94.9 & -0.000 & 0.003 & 0.004 & 96.6 \\
\hline & & $\beta_{\sigma, 2}$ & 0.08 & 0.001 & 0.008 & 0.009 & 93.6 & 0.000 & 0.006 & 0.006 & 94.7 \\
\hline & & $\beta_{\xi, 0}$ & 0.20 & -0.006 & 0.051 & 0.055 & 95.3 & -0.008 & 0.036 & 0.037 & 94.1 \\
\hline
\end{tabular}


TABLE 3

Bias, empirical standard errors (ESE), average standard errors (ASE) from the sandwich estimator, and empirical coverage percentage (CP) of $95 \%$ confidence intervals for the model parameters (Par) from the two-step approach based on 1000 replicates for the geometric Gaussian model.

\begin{tabular}{|c|c|c|c|c|c|c|c|c|c|c|c|}
\hline \multirow[b]{2}{*}{ Dep } & \multirow[b]{2}{*}{$S$} & \multirow[b]{2}{*}{ Par } & \multirow[b]{2}{*}{ True } & \multicolumn{4}{|c|}{$n=50$} & \multicolumn{4}{|c|}{$n=100$} \\
\hline & & & & bias & ESE & ASE & $\mathrm{CP}$ & bias & ESE & ASE & $\mathrm{CP}$ \\
\hline \multirow[t]{16}{*}{ W } & \multirow[t]{8}{*}{25} & $\alpha$ & 25.20 & -0.386 & 5.830 & 5.142 & 86.5 & -0.372 & 3.992 & 3.831 & 90.5 \\
\hline & & $\beta_{\mu, 0}$ & 15.00 & -0.017 & 0.256 & 0.257 & 94.8 & -0.021 & 0.174 & 0.182 & 95.3 \\
\hline & & $\beta_{\mu, 1}$ & -0.20 & 0.000 & 0.011 & 0.011 & 95.7 & 0.000 & 0.008 & 0.008 & 95.6 \\
\hline & & $\beta_{\mu, 2}$ & 0.25 & -0.000 & 0.012 & 0.012 & 94.4 & -0.000 & 0.008 & 0.009 & 94.9 \\
\hline & & $\beta_{\sigma, 0}$ & 4.00 & 0.017 & 0.206 & 0.199 & 93.0 & 0.022 & 0.146 & 0.143 & 93.7 \\
\hline & & $\beta_{\sigma, 1}$ & -0.04 & -0.000 & 0.006 & 0.006 & 95.5 & -0.000 & 0.004 & 0.004 & 95.3 \\
\hline & & $\beta_{\sigma, 2}$ & 0.08 & 0.000 & 0.007 & 0.007 & 95.1 & 0.001 & 0.005 & 0.005 & 95.1 \\
\hline & & $\beta_{\xi, 0}$ & 0.20 & -0.010 & 0.025 & 0.023 & 87.7 & -0.009 & 0.018 & 0.017 & 87.7 \\
\hline & \multirow[t]{8}{*}{50} & $\alpha$ & 25.20 & -0.456 & 5.471 & 4.851 & 87.7 & -0.496 & 3.966 & 3.622 & 89.0 \\
\hline & & $\beta_{\mu, 0}$ & 15.00 & -0.007 & 0.255 & 0.249 & 93.5 & -0.020 & 0.177 & 0.176 & 94.1 \\
\hline & & $\beta_{\mu, 1}$ & -0.20 & -0.000 & 0.010 & 0.010 & 94.3 & 0.000 & 0.007 & 0.007 & 93.9 \\
\hline & & $\beta_{\mu, 2}$ & 0.25 & -0.000 & 0.011 & 0.011 & 95.1 & -0.000 & 0.008 & 0.008 & 93.6 \\
\hline & & $\beta_{\sigma, 0}$ & 4.00 & 0.024 & 0.204 & 0.193 & 93.9 & 0.013 & 0.143 & 0.137 & 94.0 \\
\hline & & $\beta_{\sigma, 1}$ & -0.04 & -0.000 & 0.005 & 0.005 & 94.9 & -0.000 & 0.004 & 0.004 & 94.5 \\
\hline & & $\beta_{\sigma, 2}$ & 0.08 & 0.001 & 0.006 & 0.006 & 94.6 & 0.000 & 0.004 & 0.004 & 94.4 \\
\hline & & $\beta_{\xi, 0}$ & 0.20 & -0.009 & 0.024 & 0.022 & 86.3 & -0.010 & 0.017 & 0.016 & 84.0 \\
\hline \multirow[t]{16}{*}{ M } & \multirow[t]{8}{*}{25} & $\alpha$ & 135.20 & 0.432 & 28.786 & 28.622 & 93.7 & 0.523 & 20.355 & 20.399 & 94.7 \\
\hline & & $\beta_{\mu, 0}$ & 15.00 & -0.042 & 0.371 & 0.392 & 95.1 & -0.015 & 0.267 & 0.276 & 95.4 \\
\hline & & $\beta_{\mu, 1}$ & -0.20 & 0.000 & 0.011 & 0.011 & 95.6 & 0.000 & 0.007 & 0.008 & 95.4 \\
\hline & & $\beta_{\mu, 2}$ & 0.25 & -0.001 & 0.012 & 0.013 & 95.5 & -0.000 & 0.009 & 0.009 & 94.6 \\
\hline & & $\beta_{\sigma, 0}$ & 4.00 & 0.009 & 0.306 & 0.314 & 94.3 & 0.032 & 0.218 & 0.222 & 96.5 \\
\hline & & $\beta_{\sigma, 1}$ & -0.04 & -0.000 & 0.006 & 0.006 & 95.1 & -0.000 & 0.004 & 0.004 & 95.1 \\
\hline & & $\beta_{\sigma, 2}$ & 0.08 & 0.000 & 0.008 & 0.008 & 95.8 & 0.001 & 0.006 & 0.006 & 95.1 \\
\hline & & $\beta_{\xi, 0}$ & 0.20 & -0.012 & 0.040 & 0.039 & 91.5 & -0.009 & 0.028 & 0.028 & 92.5 \\
\hline & \multirow[t]{8}{*}{50} & $\alpha$ & 135.20 & -1.788 & 28.124 & 27.524 & 92.2 & -0.101 & 19.735 & 19.896 & 94.0 \\
\hline & & $\beta_{\mu, 0}$ & 15.00 & -0.025 & 0.376 & 0.390 & 95.5 & -0.017 & 0.271 & 0.273 & 93.7 \\
\hline & & $\beta_{\mu, 1}$ & -0.20 & -0.000 & 0.010 & 0.010 & 96.2 & -0.000 & 0.007 & 0.007 & 94.9 \\
\hline & & $\beta_{\mu, 2}$ & 0.25 & -0.001 & 0.012 & 0.013 & 94.9 & -0.000 & 0.008 & 0.009 & 95.0 \\
\hline & & $\beta_{\sigma, 0}$ & 4.00 & 0.008 & 0.317 & 0.310 & 94.2 & 0.019 & 0.218 & 0.219 & 94.4 \\
\hline & & $\beta_{\sigma, 1}$ & -0.04 & -0.000 & 0.006 & 0.006 & 96.8 & -0.000 & 0.004 & 0.004 & 95.2 \\
\hline & & $\beta_{\sigma, 2}$ & 0.08 & 0.000 & 0.008 & 0.008 & 95.0 & 0.000 & 0.006 & 0.006 & 95.1 \\
\hline & & $\beta_{\xi, 0}$ & 0.20 & -0.014 & 0.040 & 0.038 & 90.1 & -0.011 & 0.028 & 0.027 & 91.0 \\
\hline \multirow[t]{16}{*}{ S } & \multirow[t]{8}{*}{25} & $\alpha$ & 1252.00 & 6.615 & 262.957 & 284.670 & 94.9 & 7.647 & 182.119 & 196.260 & 95.8 \\
\hline & & $\beta_{\mu, 0}$ & 15.00 & -0.058 & 0.450 & 0.476 & 95.2 & -0.049 & 0.306 & 0.329 & 95.4 \\
\hline & & $\beta_{\mu, 1}$ & -0.20 & 0.001 & 0.007 & 0.008 & 94.9 & 0.000 & 0.005 & 0.005 & 95.2 \\
\hline & & $\beta_{\mu, 2}$ & 0.25 & -0.001 & 0.011 & 0.011 & 95.5 & -0.001 & 0.007 & 0.008 & 95.1 \\
\hline & & $\beta_{\sigma, 0}$ & 4.00 & 0.013 & 0.368 & 0.393 & 94.5 & 0.022 & 0.251 & 0.269 & 96.2 \\
\hline & & $\beta_{\sigma, 1}$ & -0.04 & -0.000 & 0.005 & 0.005 & 94.8 & -0.000 & 0.003 & 0.004 & 96.0 \\
\hline & & $\beta_{\sigma, 2}$ & 0.08 & 0.000 & 0.008 & 0.009 & 94.1 & 0.000 & 0.006 & 0.006 & 96.4 \\
\hline & & $\beta_{\xi, 0}$ & 0.20 & -0.014 & 0.052 & 0.055 & 93.7 & -0.012 & 0.036 & 0.038 & 93.7 \\
\hline & \multirow[t]{8}{*}{50} & $\alpha$ & 1252.00 & 10.638 & 271.570 & 284.330 & 95.1 & 5.107 & 187.077 & 194.240 & 95.4 \\
\hline & & $\beta_{\mu, 0}$ & 15.00 & -0.077 & 0.446 & 0.476 & 93.6 & -0.046 & 0.318 & 0.329 & 94.4 \\
\hline & & $\beta_{\mu, 1}$ & -0.20 & 0.001 & 0.007 & 0.007 & 95.4 & 0.000 & 0.005 & 0.005 & 94.3 \\
\hline & & $\beta_{\mu, 2}$ & 0.25 & -0.002 & 0.010 & 0.011 & 93.9 & -0.001 & 0.007 & 0.008 & 94.8 \\
\hline & & $\beta_{\sigma, 0}$ & 4.00 & 0.008 & 0.374 & 0.394 & 95.3 & 0.021 & 0.260 & 0.271 & 95.4 \\
\hline & & $\beta_{\sigma, 1}$ & -0.04 & -0.000 & 0.005 & 0.005 & 95.0 & -0.000 & 0.004 & 0.004 & 94.8 \\
\hline & & $\beta_{\sigma, 2}$ & 0.08 & 0.000 & 0.008 & 0.009 & 94.6 & 0.000 & 0.006 & 0.006 & 95.8 \\
\hline & & $\beta_{\xi, 0}$ & 0.20 & -0.013 & 0.053 & 0.055 & 94.1 & -0.012 & 0.036 & 0.038 & 94.0 \\
\hline
\end{tabular}


TABLE 4

Relative efficiency (\%) in MSE of joint and individual 50-year return levels for the pairwise likelihood approach using block maxima data relative to the two-step approach for Smith, Schlather, and geometric Gaussian models. The three sites used were $s_{1}=(10,10), s_{2}=(10,11)$ and $s_{3}=(10,0)$.

\begin{tabular}{|c|c|c|c|c|c|c|c|c|c|c|c|c|c|c|c|c|c|c|}
\hline \multirow{3}{*}{$\begin{array}{l}\text { Dep } \\
n \\
S\end{array}$} & \multicolumn{6}{|c|}{ W } & \multicolumn{6}{|c|}{ M } & \multicolumn{6}{|c|}{ S } \\
\hline & \multicolumn{2}{|c|}{20} & \multicolumn{2}{|c|}{50} & \multicolumn{2}{|c|}{100} & \multicolumn{2}{|c|}{20} & \multicolumn{2}{|c|}{50} & \multicolumn{2}{|c|}{100} & \multicolumn{2}{|c|}{20} & \multicolumn{2}{|c|}{50} & \multicolumn{2}{|c|}{100} \\
\hline & 25 & 50 & 25 & 50 & 25 & 50 & 25 & 50 & 25 & 50 & 25 & 50 & 25 & 50 & 25 & 50 & 25 & 50 \\
\hline \multicolumn{19}{|l|}{ Smith } \\
\hline$\left(s_{1}, s_{2}\right)$ & 58 & 62 & 61 & 61 & 60 & 60 & 72 & 75 & 75 & 71 & 80 & 72 & 103 & 101 & 91 & 98 & 97 & 97 \\
\hline$\left(s_{1}, s_{3}\right)$ & 67 & 72 & 72 & 73 & 72 & 70 & 75 & 78 & 77 & 75 & 83 & 75 & 104 & 101 & 91 & 98 & 97 & 97 \\
\hline$s_{1}$ & 56 & 61 & 60 & 61 & 60 & 60 & 72 & 74 & 75 & 71 & 80 & 72 & 103 & 101 & 91 & 98 & 97 & 97 \\
\hline$s_{2}$ & 56 & 60 & 60 & 60 & 60 & 59 & 71 & 74 & 75 & 71 & 79 & 71 & 103 & 101 & 91 & 98 & 97 & 97 \\
\hline$s_{3}$ & 59 & 62 & 64 & 64 & 61 & 64 & 76 & 76 & 75 & 75 & 82 & 75 & 106 & 102 & 91 & 98 & 97 & 97 \\
\hline \multicolumn{19}{|l|}{ Schlather } \\
\hline$\left(s_{1}, s_{2}\right)$ & 76 & 82 & 78 & 82 & 78 & 75 & 82 & 78 & 84 & 80 & 79 & 80 & 95 & 104 & 90 & 90 & 82 & 83 \\
\hline$\left(s_{1}, s_{3}\right)$ & 84 & 91 & 86 & 89 & 85 & 84 & 87 & 83 & 87 & 83 & 83 & 84 & 95 & 104 & 90 & 90 & 83 & 84 \\
\hline$s_{1}$ & 71 & 77 & 74 & 78 & 73 & 71 & 80 & 76 & 83 & 78 & 77 & 79 & 95 & 104 & 90 & 89 & 82 & 83 \\
\hline$s_{2}$ & 71 & 77 & 74 & 78 & 73 & 71 & 80 & 76 & 82 & 78 & 77 & 79 & 95 & 104 & 90 & 89 & 82 & 83 \\
\hline$s_{3}$ & 70 & 78 & 74 & 78 & 73 & 72 & 83 & 77 & 83 & 78 & 78 & 78 & 95 & 105 & 89 & 89 & 82 & 82 \\
\hline \multicolumn{19}{|c|}{ Geometric Gaussian } \\
\hline$\left(s_{1}, s_{2}\right)$ & 64 & 69 & 68 & 69 & 69 & 72 & 85 & 82 & 82 & 84 & 84 & 89 & 91 & 87 & 93 & 96 & 94 & 94 \\
\hline$\left(s_{1}, s_{3}\right)$ & 62 & 68 & 67 & 68 & 67 & 72 & 82 & 81 & 79 & 81 & 80 & 86 & 98 & 99 & 99 & 101 & 99 & 97 \\
\hline$s_{1}$ & 64 & 69 & 68 & 69 & 69 & 74 & 82 & 80 & 78 & 81 & 80 & 85 & 95 & 110 & 97 & 99 & 97 & 94 \\
\hline$s_{2}$ & 64 & 69 & 68 & 69 & 69 & 73 & 81 & 80 & 78 & 81 & 80 & 85 & 95 & 109 & 97 & 99 & 97 & 94 \\
\hline$s_{3}$ & 65 & 71 & 70 & 71 & 71 & 76 & 83 & 82 & 78 & 84 & 81 & 87 & 96 & 111 & 97 & 100 & 96 & 95 \\
\hline
\end{tabular}

We considered $\nu \in\{1,2\}$ for reasons described later. For a given $\nu$, a nonlinear least square fit in the range of $[0,40]$ is again employed to give the value of $\alpha$ such that the bivariate extremal coefficient as a function of distance matches closely that from the Smith model. For $\nu=1$, the values of $\alpha$ were found to be 5.2, 24.3, 242.9 for weak, moderate, and strong dependence, respectively. For $\nu=2$, the corresponding values were $7.9,41.6$, 398.3 .

In each season, $m$ independent realizations were generated a centered $t$ process with $\nu$ d.f. and a dispersion function $\rho$. The convergence of the dependence structure to extremal- $t$ is ensured following Opitz (2013). For the marginal maxima to converge to the desired marginal GEV distributions, these realizations had to be appropriately standardized by subtracting $b_{m}$ and then dividing by $a_{m}$. In this setting, we used $b_{m}=0$ and $a_{m}=m^{1 / \nu} c_{\nu}^{1 / \nu}$, 
72

where

$$
c_{\nu}=\frac{v^{\frac{\nu-1}{2}} \Gamma\left(\frac{\nu+1}{2}\right)}{\sqrt{\nu \pi} \Gamma\left(\frac{\nu}{2}\right)} .
$$

Note that this expression corrects the misprints in the definition of $c_{\nu}$ on the second line of section 2.4.2 in Opitz (2013). After the standardization, the transformed process has $\nu$-Fréchet marginal distributions with distribution function $F(z ; \nu)=\exp \left(-z^{-\nu}\right)$ for $z>0$, as $m \rightarrow \infty$. These $\nu$-Fréchet variates were then transformed to give daily records such that the maxima of the transformed variables follow the desired GEV distribution.

2.3. Choosing $\nu$ via Goodness-of-Fit Tests. Three aspects of the convergence affect the model specification of the two-step approach, mis-specification of any of which may lead to bias. First, the correctness of the extremal- $t$ model depends on the convergence of the $t$ processes to the extremal- $t$ process. Second, the correctness of the marginal GEV model specification depends on convergence of the marginal distributions of the $t$ process to the $\nu$-Fréchet distribution. Third, the correctness of the point process model specification also depends on the convergence of the exceedances over the threshold to the GPD distribution with appropriate parameters. The pairwise likelihood approach only depends on the first two.

How large do we need the block size $m$ and the threshold $u$ to be for the limiting distribution to approximate well in the three aspects? We considered three sets of goodness-of-fit tests for the limiting distribution with various combinations of $m, u$, and $\nu$. The first was the goodness-of-fit test for bivariate extremal- $t$ for pairs using the method of Genest et al. (2011). The test statistic summarizes the difference between a nonparametric and a parametric estimator of the Pickands dependence function. Approximate $\mathrm{p}$-values were obtained from a parametric bootstrap procedure in $\mathrm{R}$ package copula (Kojadinovic and Yan, 2010). The second was the KolmogorovSmirnov test for the goodness-of-fit of the marginal $\nu$-Fréchet distribution on the standardized $t$ variates. The third was the Kolmogorov-Smirnov test for the marginal GPD with appropriate parameters on the exceedances over the threshold. We did the first test on pairs $\left(s_{1}, s_{2}\right)$ and $\left(s_{1}, s_{3}\right)$, and the second and third test for site $s_{1}$, where $s_{1}=(10,10), s_{2}=(10,11)$, and $s_{3}=(0,10)$.

We experimented with $\nu \in\{1,2,3,5\}, m \in\{100,200,500,1000,2000\}$. For testing the GPD of the exceedances, we used the $95 \%, 98 \%$ and $99 \%$ percentiles as the threshold $u$. After transforming the data to the scale of unit-Fréchet, the specific GPD distribution is $\operatorname{GPD}(u, u, 1)$. The sample size $n$ was fixed at 100 . The range parameter $\alpha$ was fixed at 24.3, corresponding to 
TABLE 5

Empirical rejection percentage from 1000 replicates in each scenario for testing bivariate and univariate extremal-t distributions.

\begin{tabular}{rrrrrrrr}
\hline$\nu$ & $m$ & $\left(s_{1}, s_{2}\right)$ & $\left(s_{1}, s_{3}\right)$ & GEV & GPD $(95 \%)$ & GPD $(98 \%)$ & GPD $(99 \%)$ \\
\hline 1 & 100 & 3.3 & 4.3 & 4.5 & 3.7 & 3.6 & 4.7 \\
& 200 & 4.3 & 3.3 & 4.3 & 6.4 & 4.6 & 4.1 \\
& 500 & 3.8 & 4.5 & 4.2 & 6.2 & 5.6 & 4.7 \\
& 1000 & 3.8 & 3.7 & 4.7 & 7.2 & 4.7 & 5.8 \\
& 2000 & 4.0 & 4.3 & 5.1 & 9.7 & 4.7 & 6.0 \\
2 & 100 & 5.4 & 5.4 & 4.4 & 48.6 & 7.5 & 5.5 \\
& 200 & 3.6 & 4.9 & 5.5 & 76.2 & 9.2 & 6.6 \\
& 500 & 5.2 & 4.5 & 4.0 & 98.8 & 17.6 & 5.9 \\
& 1000 & 4.0 & 3.0 & 5.7 & 100.0 & 30.0 & 6.1 \\
& 2000 & 5.2 & 4.3 & 4.5 & 100.0 & 50.7 & 11.1 \\
3 & 100 & 4.1 & 5.9 & 20.1 & 99.7 & 28.5 & 8.7 \\
& 200 & 3.9 & 4.1 & 13.1 & 100.0 & 53.7 & 14.3 \\
& 500 & 4.5 & 5.0 & 7.1 & 100.0 & 91.1 & 32.4 \\
& 1000 & 4.5 & 5.0 & 6.3 & 100.0 & 99.7 & 53.7 \\
& 2000 & 4.8 & 4.3 & 4.9 & 100.0 & 100.0 & 81.1 \\
5 & 100 & 4.5 & 5.7 & 100.0 & 100.0 & 97.6 & 52.1 \\
& 200 & 4.5 & 5.5 & 98.6 & 100.0 & 100.0 & 80.5 \\
& 500 & 4.4 & 5.3 & 80.4 & 100.0 & 100.0 & 100.0 \\
& 1000 & 4.6 & 4.4 & 59.8 & 100.0 & 100.0 & 100.0 \\
& 2000 & 4.2 & 4.2 & 36.7 & 100.0 & 100.0 & 100.0 \\
\hline
\end{tabular}

the moderate dependence level for $\nu=1$. Note that although the extremal- $t$ process becomes the Schlather model when $\nu=1$, this simulation setting is still different from the one with data generated from the Schlather model, because the model specification for the Schlather setting was exact.

Table 5 shows the empirical rejection rates of these tests with significance level $5 \%$ from 1000 replicates. The levels of the bivariate goodness-of-fit tests for both pairs appear to be maintained even for $m=100$, suggesting that, surprisingly, the convergence to the extremal- $t$ copulas occurs very fast. For the GEV distribution of marginal block maxima, the larger $\nu$ is, the larger $m$ is needed for the limiting distribution to pass the test. At $\nu=5$, even an $m=2000$ was not enough for the convergence. For testing the GPD, larger rejection rates were observed as $\nu$ increases or as $u$ decreases, and both patterns were as expected. For fixed $\nu$, larger $m$ leads to large rejection rate, which is explained by the increased sample size of the exceedances. For fixed $\nu$ and $m$, the percentage of rejections generally decreases as the threshold increases, which may also be explained by the decreased sample size of the exceedances.

These results suggest that the three aspects of the convergence were not 
alarming for $\nu=1$, but the GPD approximation of the exceedances may have problems. Therefore, we chose $\nu \in\{1,2\}$ to study the two-step method and we expect bias when $\nu=2$.

2.3.1. Relative Efficiency in Estimating the Model Parameters. Table 6 summarizes the relative efficiency of the pairwise likelihood method with the two-step method as the reference in estimating model parameters for $\nu \in\{1,2\}$. The two-step method is the clear winner for $\nu=1$. For $\nu=2$, the two-step method is still more efficient in most entries but lost to the pairwise likelihood method in a few entries. For example, consider the scenario with relative efficiency of $210 \%$ (for $\beta_{\xi, 0}$ with $n=200$ and $S=25$ under weak dependence). For the two-step approach, the MSE was 0.00303 which was decomposed into squared bias was 0.00263 and variance 0.00040 ; for the pairwise likelihood approach, the MSE was 0.00145 , which was decomposed into squared bias 0.00025 and variance 0.00120 . Although the variance for two-step estimate is much smaller than the variance for pairwise likelihood estimate, its much bigger bias led to a much bigger MSE compared to the pairwise likelihood estimate. The bias is explained by the goodness-of-fit results for the GPD on the exceedances in Table 5.

2.3.2. Relative Efficiency in Estimating Return Levels. Table 7 is the counterpart of Table 4, summarizing the relative efficiency of the pairwise likelihood approach using block maxima only with the two-step approach as the reference in estimating joint and individual return levels.

3. More Figures for the California Precipitation Analysis. Figure 1 presents the pairwise extremal coefficients diagnosis based on madogram.

Figure 2 presents the empirical quantile versus model quantiles for the annual maxima of fours subsets of sites grouped by their latitude.

Figure 3 presents the map of the standardized SOI coefficients (estimate divided by standard error) in the location parameter of the GEV models. They are the $z$-scores that can be used to test the null hypotheses that the corresponding coefficient at each site is zero.

Figure 4 presents the box plots of the ratio of the standard errors of the four parameter estimates from the two-step method and that from the pairwise likelihood method across 35 site.

\section{References.}

Genest, C., Kojadinovic, I., Nešlehová, J. and Yan, J. (2011). A Goodness-of-fit Test for Bivariate Extreme-value Copulas. Bernoulli 17 253-275. 
TABLE 6

Relative efficiency (\%) in mean squared error of model parameter estimates for the pairwise likelihood approach using block maxima data relative to the two-step approach for the extremal-t processes.

\begin{tabular}{|c|c|c|c|c|c|c|c|c|c|c|c|c|c|c|c|c|c|c|}
\hline \multirow[b]{2}{*}{ Dep } & \multirow[b]{2}{*}{$n$} & \multirow[b]{2}{*}{$S$} & \multicolumn{8}{|c|}{ Extremal- $t_{\nu=1}$} & \multicolumn{8}{|c|}{ Extremal- $t_{\nu=2}$} \\
\hline & & & $\alpha$ & $\beta_{\mu, 0}$ & $\beta_{\mu, 1}$ & $\beta_{\mu, 2}$ & $\beta_{\sigma, 0}$ & $\beta_{\sigma, 1}$ & $\beta_{\sigma, 2}$ & $\overline{\beta_{\xi, 0}}$ & $\alpha$ & $\beta_{\mu, 0}$ & $\beta_{\mu, 1}$ & $\beta_{\mu, 2}$ & $\beta_{\sigma, 0}$ & $\beta_{\sigma, 1}$ & $\beta_{\sigma, 2}$ & $\overline{\beta_{\xi, 0}}$ \\
\hline \multirow[t]{6}{*}{ W } & 20 & 25 & 99 & 73 & 61 & 66 & 90 & 37 & 57 & 34 & 87 & 76 & 56 & 55 & 90 & 27 & 33 & 72 \\
\hline & & 50 & 103 & 64 & 64 & 62 & 91 & 43 & 50 & 32 & 85 & 77 & 57 & 59 & 101 & 28 & 41 & 76 \\
\hline & 50 & 25 & 97 & 64 & 62 & 61 & 86 & 36 & 44 & 34 & 95 & 75 & 54 & 62 & 98 & 25 & 37 & 131 \\
\hline & & 50 & 100 & 70 & 62 & 68 & 87 & 41 & 52 & 41 & 94 & 81 & 58 & 64 & 101 & 33 & 44 & 136 \\
\hline & 100 & 25 & 96 & 70 & 63 & 63 & 90 & 36 & 50 & 39 & 107 & 67 & 52 & 55 & 108 & 29 & 43 & 210 \\
\hline & & 50 & 103 & 73 & 61 & 62 & 97 & 37 & 59 & 39 & 102 & 71 & 60 & 59 & 114 & 32 & 49 & 207 \\
\hline \multirow[t]{6}{*}{$\mathrm{M}$} & 20 & 25 & 88 & 67 & 69 & 63 & 80 & 40 & 52 & 36 & 91 & 66 & 57 & 53 & 82 & 29 & 37 & 71 \\
\hline & & 50 & 95 & 65 & 60 & 67 & 84 & 48 & 55 & 39 & 81 & 63 & 53 & 59 & 81 & 31 & 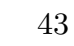 & 64 \\
\hline & 50 & 25 & 93 & 60 & 64 & 69 & 78 & 37 & 51 & 38 & 87 & 65 & 56 & 58 & 85 & 31 & 41 & 98 \\
\hline & & 50 & 90 & 68 & 70 & 69 & 83 & 42 & 54 & 40 & 86 & 66 & 60 & 5 & 90 & 3 & 43 & 100 \\
\hline & 100 & 25 & 90 & 62 & 68 & 64 & 82 & 44 & 57 & 40 & 92 & 68 & 58 & 60 & 97 & 34 & 52 & 153 \\
\hline & & 50 & 90 & 69 & 65 & 66 & 82 & 43 & 55 & 44 & 91 & 62 & 59 & 58 & 92 & 35 & 52 & 152 \\
\hline \multirow[t]{6}{*}{$\mathrm{S}$} & 20 & 25 & 82 & 52 & 81 & 62 & 60 & 60 & 58 & 43 & 81 & 59 & 80 & 65 & 68 & 56 & 57 & 70 \\
\hline & & 50 & 84 & 51 & 70 & 58 & 68 & 57 & 62 & 40 & 80 & 54 & 63 & 56 & 64 & 45 & 53 & 66 \\
\hline & 50 & 25 & 88 & 58 & 89 & 68 & 70 & 62 & 65 & 42 & 81 & 59 & 64 & 63 & 72 & 55 & 58 & 96 \\
\hline & & 50 & 83 & 57 & 79 & 69 & 64 & 61 & 65 & 45 & 77 & 59 & 68 & 62 & 74 & 52 & 64 & 99 \\
\hline & 100 & 25 & 85 & 56 & 74 & 65 & 63 & 59 & 58 & 48 & 73 & 58 & 61 & 61 & 77 & 56 & 65 & 129 \\
\hline & & 50 & 87 & 59 & 78 & 67 & 64 & 63 & 63 & 51 & 78 & 57 & 71 & 60 & 78 & 54 & 70 & 131 \\
\hline
\end{tabular}

Heagerty, P. J. and Lele, S. R. (1998). A Composite Likelihood Approach to Binary Spatial Data. Journal of the American Statistical Association 93 1099-1111.

Heagerty, P. J. and Lumley, T. (2000). Window Subsampling of Estimating Functions with Application to Regression Models. Journal of the American Statistical Association 95 197-211.

Kojadinovic, I. and Yan, J. (2010). Modeling Multivariate Distributions with Continuous Margins Using the copula R Package. Journal of Statistical Software 34 1-20.

Opitz, T. (2013). Extremal t Processes: Elliptical Domain of Attraction and a Spectral Representation. Journal of Multivariate Analysis 122 409-413. 
TABLE 7

Relative efficiency (\%) in MSE of joint and individual 50-year return levels for the pairwise likelihood approach using block maxima data relative to the two-step approach for two extremal-t models. The three sites used were $s_{1}=(10,10), s_{2}=(10,11)$ and $s_{3}=(10,0)$.

\begin{tabular}{|c|c|c|c|c|c|c|c|c|c|c|c|c|c|c|c|c|c|c|}
\hline \multirow{3}{*}{$\begin{array}{l}\text { Dep } \\
n \\
S\end{array}$} & \multicolumn{6}{|c|}{ W } & \multicolumn{6}{|c|}{ M } & \multicolumn{6}{|c|}{$\mathrm{S}$} \\
\hline & \multicolumn{2}{|c|}{20} & \multicolumn{2}{|c|}{50} & \multicolumn{2}{|c|}{100} & \multicolumn{2}{|c|}{20} & \multicolumn{2}{|c|}{50} & \multicolumn{2}{|c|}{100} & \multicolumn{2}{|c|}{20} & \multicolumn{2}{|c|}{50} & \multicolumn{2}{|c|}{100} \\
\hline & 25 & 50 & 25 & 50 & 25 & 50 & 25 & 50 & 25 & 50 & 25 & 50 & 25 & 50 & 25 & 50 & 25 & 50 \\
\hline \multicolumn{19}{|c|}{ Extremal- $t_{\nu=1}$} \\
\hline$\left(s_{1}, s_{2}\right)$ & 73 & & 76 & 81 & 82 & 82 & 70 & 81 & 79 & 76 & 76 & 83 & 103 & 92 & 84 & 81 & & 86 \\
\hline$\left(s_{1}, s_{3}\right)$ & 81 & 80 & 84 & 88 & 89 & 90 & 74 & 86 & 82 & 80 & 80 & 86 & 103 & 93 & 85 & 82 & 86 & 86 \\
\hline$s_{1}$ & 69 & 67 & 71 & 77 & 77 & 77 & 68 & 79 & 77 & 74 & 75 & 81 & 102 & 91 & 84 & 81 & 85 & 85 \\
\hline$s_{2}$ & 69 & & 71 & 77 & 77 & 76 & 68 & 79 & 77 & 74 & 74 & 81 & 102 & 91 & 84 & 81 & 85 & 85 \\
\hline$s_{3}$ & 69 & 69 & 72 & 79 & 77 & 77 & 69 & 80 & 76 & 75 & 75 & 82 & 103 & 92 & 84 & 81 & 85 & 85 \\
\hline \multicolumn{19}{|c|}{ Extremal- $t_{\nu=2}$} \\
\hline$\left(s_{1}, s_{2}\right)$ & 71 & 68 & 87 & 89 & 108 & 106 & 73 & 68 & 80 & 77 & 98 & 96 & 89 & 86 & 87 & 85 & 91 & 93 \\
\hline$\left(s_{1}, s_{3}\right)$ & 77 & 74 & 87 & 88 & 101 & 99 & 77 & 72 & 81 & 79 & 97 & 95 & 89 & 86 & 88 & 86 & 90 & 93 \\
\hline$s_{1}$ & 67 & 65 & 85 & 88 & 110 & 109 & 72 & 67 & 79 & 77 & 99 & 98 & 89 & 86 & 87 & 85 & 91 & 94 \\
\hline$s_{2}$ & 66 & 65 & 85 & 88 & 109 & 109 & 72 & 67 & 79 & 77 & 99 & 97 & 89 & 86 & 87 & 85 & 91 & 94 \\
\hline$s_{3}$ & 69 & 67 & 86 & 89 & 112 & 111 & 74 & 68 & 79 & 79 & 99 & 99 & 89 & 85 & 87 & 85 & 90 & 93 \\
\hline
\end{tabular}




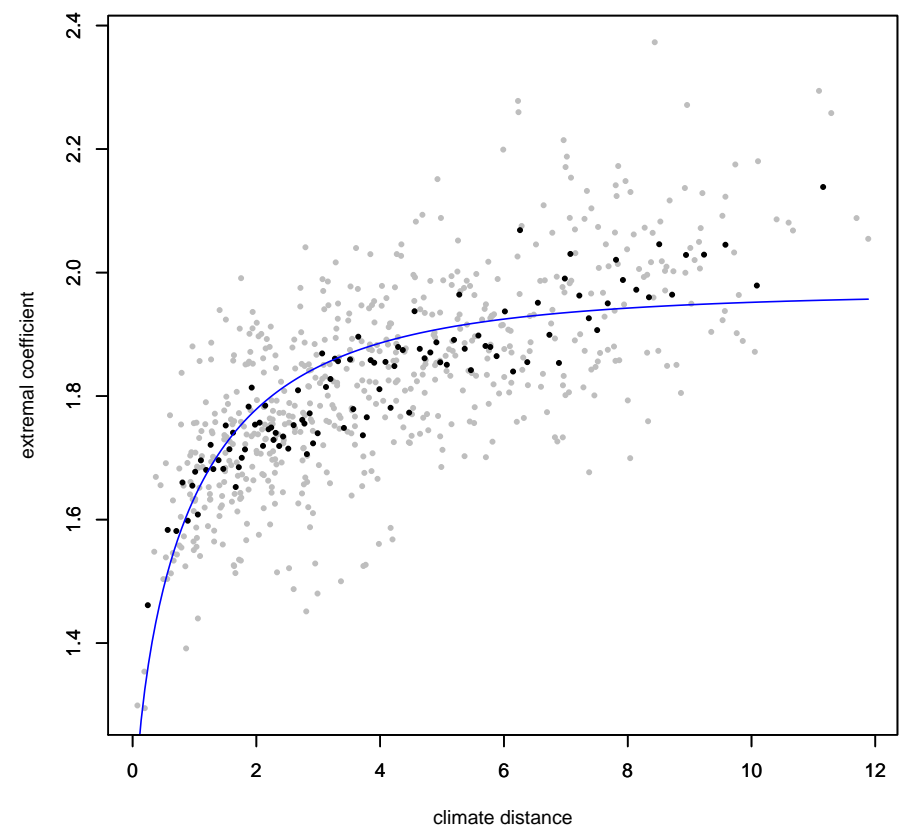

FIG 1. Pairwise extremal coefficients diagnosis. The grey dots represent pairwise madogram, the black dots represent madogram with 100 bins, and the line is the fitted extremal coefficient curve for the isotropic geometric Gaussian model. 


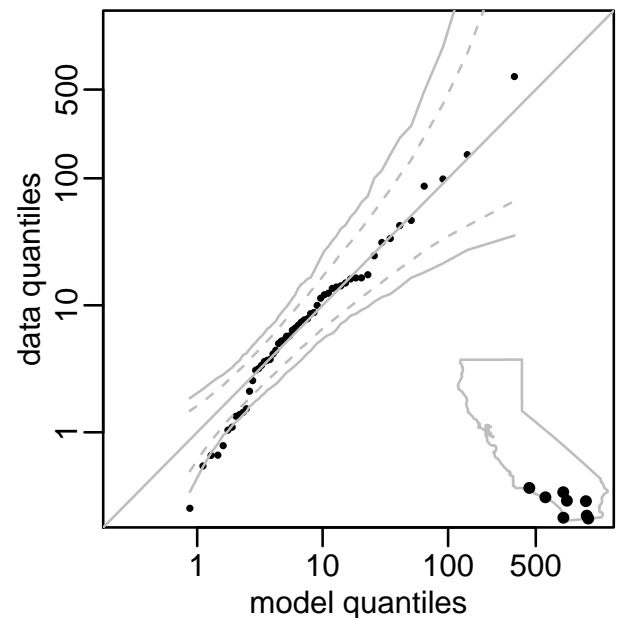

(a)

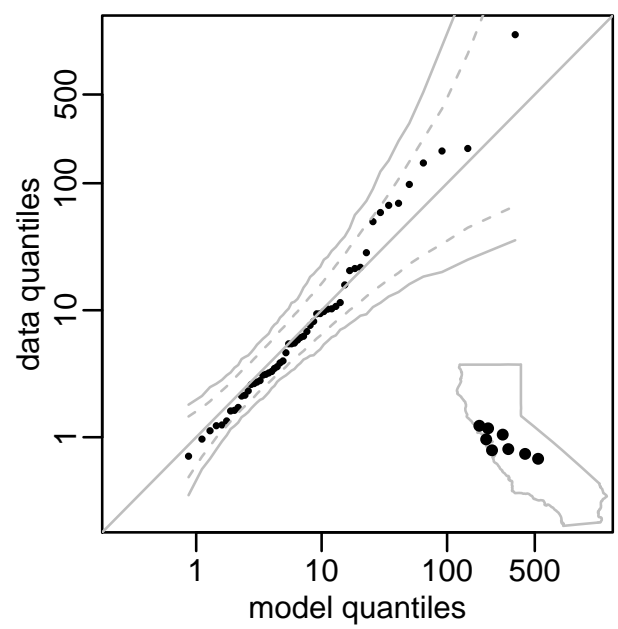

(c)

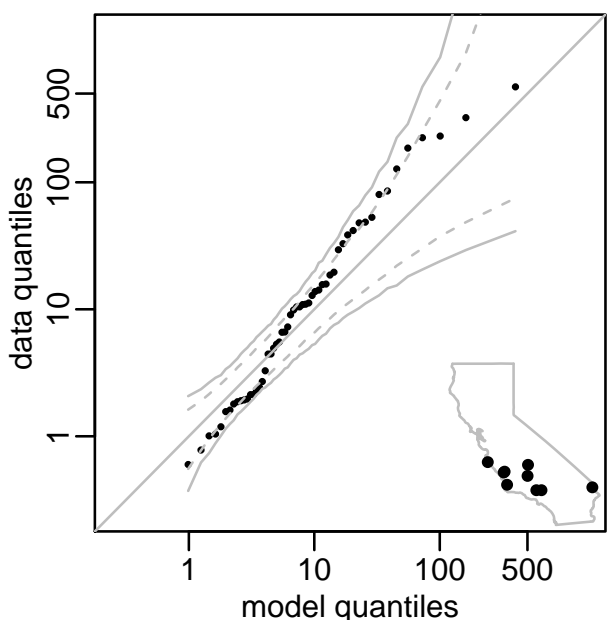

(b)

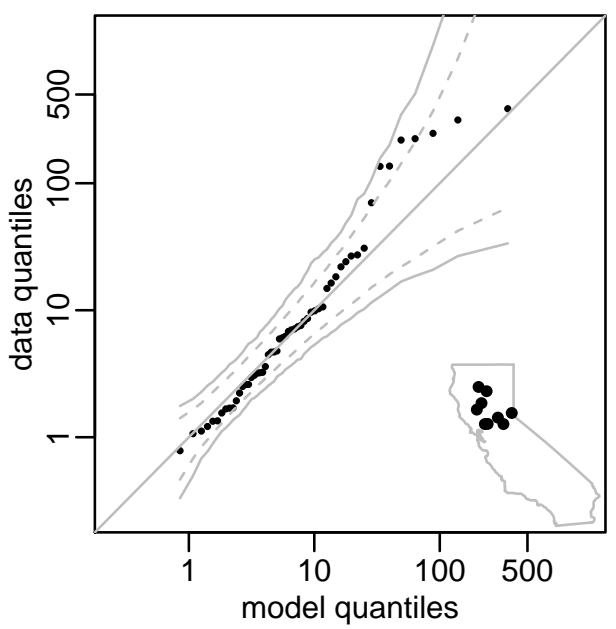

(d)

FIG 2. Comparison of empirical and model quantiles for annual maxima of four subsets of sites. The sites used in each panel are shown in its map. The the outer lines are 95\% confidence band and the inner lines are $95 \%$ pointwise confidence intervals obtained from $K=5000$ simulated realizations from the fitted model. 


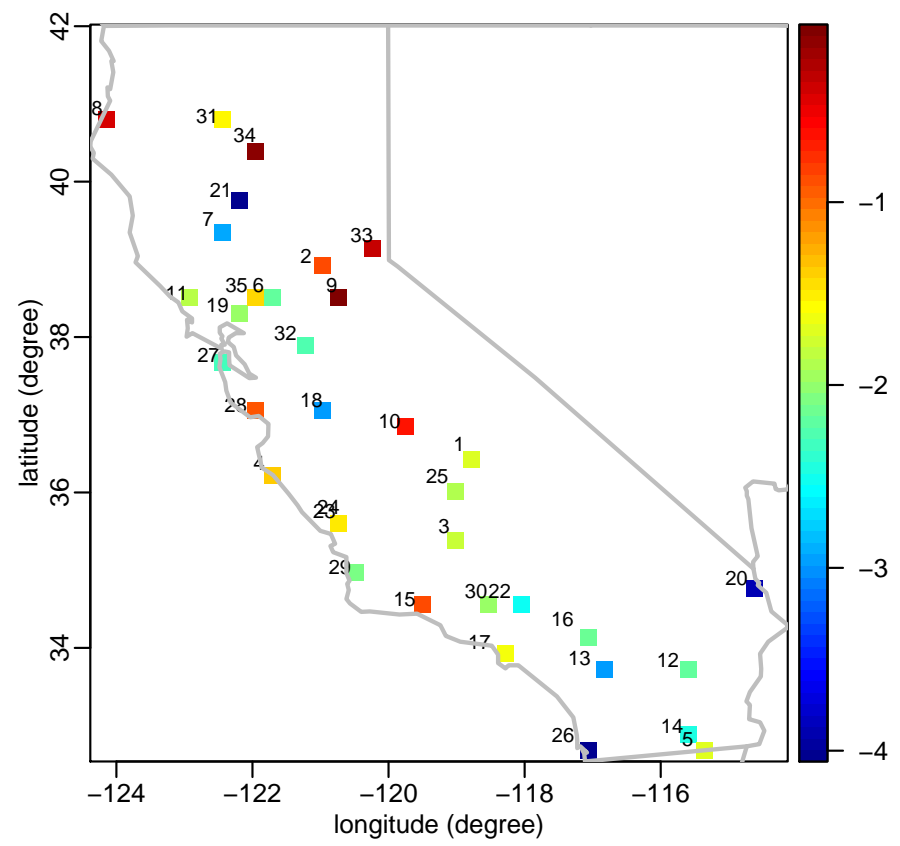

FIG 3. The standardized SOI coefficients in the location parameters at 35 sites, with all sites labeled.

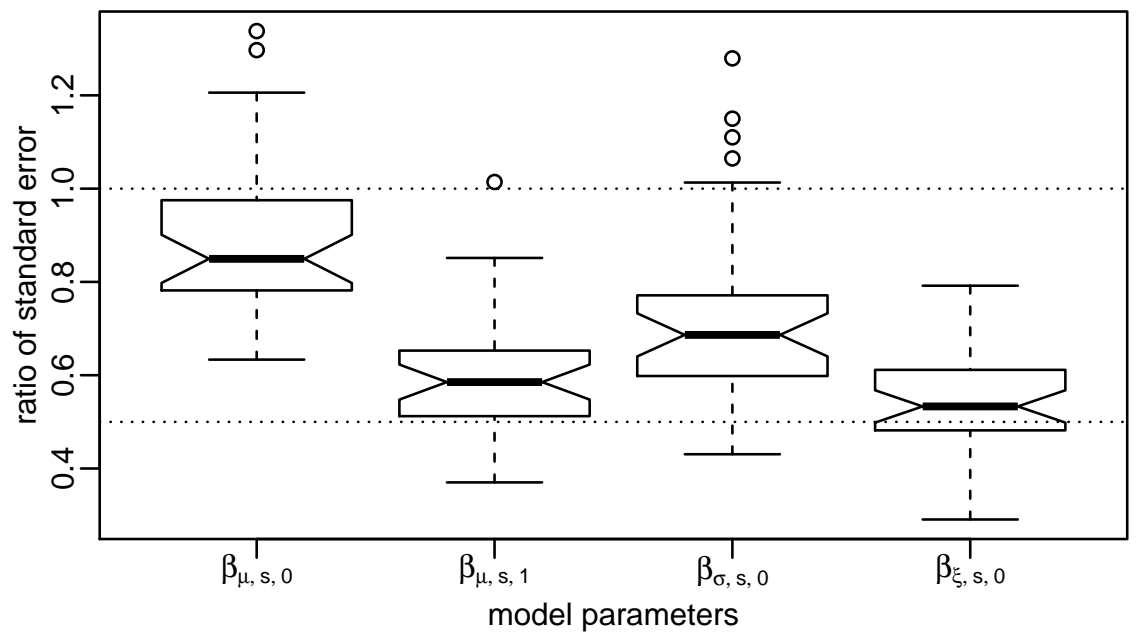

FIG 4. Box plot of the ratio of the standard errors of the four marginal parameter estimates at 35 sites, $M_{2}$ versus $M_{1}$. 